\title{
Impact of intraoperative Cone-Beam Computed Tomography use on patient satisfaction after closed nasal reduction*
}

\author{
Catrin Bruehlmann ${ }^{1,2}$, Michael Blumer ${ }^{2,3, \#}$, Michael B. Soyka ${ }^{1,2, \#}$ \\ ' University Hospital Zurich, Department of Otorhinolaryngology, Head \& Neck Surgery, Switzerland \\ 2 University of Zürich, Switzerland \\ University Hospital Zurich, Department of Oral and Maxillofacial Surgery, Switzerland
}

Rhinology Online, Vol 4: 77 - 84, 2021 http://doi.org/10.4193/RHINOL/21.018

*Received for publication:

April 15, 2020

Accepted: April 26, 2021

Published: May 4, 2021

\# Shared last authorship

\# Shared last authorship

\begin{abstract}
Background: The aim of the study was to assess, whether there is a difference in aesthetic and functional patient satisfaction between closed nasal reductions with intraoperative Cone-Beam Computed Tomography (CBCT) and without using intraoperative medical imaging.
\end{abstract}

Methods: A monocentric, retrospective cohort study of 43 patients (20 patients treated with intraoperative CBCT and 23 patients treated without intraoperative imaging) was conducted. Subjective postoperative aesthetic and functional aspects of the nose were assessed. Additionally, questions comparing the aesthetics and function of the nose before and after the accident and on the desire of revision surgery were asked.

Results: Both the SCHNOS-C and total SCHNOS score in the non-CBCT group were higher than the respective scores of the CBCTgroup. The comparison of SCHNOS-C between male subjects of the two groups showed no statistical significance. The comparison of SCHNOS-C between male and female subjects over both groups showed significantly higher scores for female subjects.

Conclusions: Patients undergoing surgery with intraoperative CBCT imaging showed better aesthetical outcomes than patients, treated without intraoperative imaging. However, the difference showed no clinical importance, so that both strategies appear to have comparable outcomes regarding postoperative aesthetics and function of the nose. Gender instead of the different strategies could contribute to the demonstrated differences. Female subjects seem to be less satisfied with the aesthetics of their nose postoperatively, potentially being more sensitive to remaining nasal deformities after surgery.

Key words: nasal bone, fracture, outcome, nasal reduction, intraoperative imaging

\section{Introduction}

Nasal fractures are the most common bony injuries, resulting from facial trauma (1), often caused by sports or traffic accidents, as well as interpersonal violence ${ }^{(2)}$. For a very long time, reduction of the fracture has been the standard therapy ${ }^{(3)}$. Closed nasal fracture reduction is performed by more than one medical department; i.e. by ENT, plastic surgery and also oral and maxillofacial surgery departments ${ }^{(4)}$.
Although the techniques in different departments are comparable, different strategies of pre- and intraoperative medical imaging are being used. A nasal fracture can be diagnosed, using Cone-Beam Computed Tomography $(\mathrm{CBCT})^{(5,6)}$, Computed Tomography and Ultrasound ${ }^{(7)}$, but mostly a nasal fracture is diagnosed without any imaging modalities, only by clinical examination of the nose.

Medical imaging cannot only be used for nasal fracture diagno- 
sis, but also for intraoperative positioning of the displaced nasal bony parts during closed nasal reduction. At the University Hospital of Zurich the Department of Otorhinolaryngology Head and Neck Surgery (ENT), as well as the Department of Oral and Maxillofacial Surgery (OMF) perform closed nasal reductions. Since July 2017 the OMF department uses intraoperative CBCT imaging during nasal reduction to confirm the correct positioning of the displaced bony elements. The ENT department performs closed nasal reduction without using any imaging modalities.

To date it is unclear whether there is a difference in postoperative aesthetic and functional patient satisfaction between the two different strategies. The aim of the present study was to assess, whether postoperative patient satisfaction differs between nasal reductions with and without intraoperative $\mathrm{CBCT}$ imaging. If so, the present study could help to standardize and optimize the treatment protocol of closed nasal fracture reductions.

\section{Materials and methods}

\section{Ethics}

This study was approved by the Swiss Ethics Committee of research involving humans (No. KEK 2020-02013) and was conducted with oral consent from every patient. The patients were fully informed about the study goals during a first phone call. Patients who were willing to participate, were given a reflection period of one week. After this period, during a second phone call, the patients were interrogated. The present study was conducted in compliance with the permission of the independent ethical commission, the current Helsinki Declaration, as well as Swiss law.

\section{Study design and population}

The project design was a monocentric, retrospective cohort study of patients with a past nasal fracture who underwent a closed nasal fracture reduction in either the ENT department or the OMF department at the University Hospital of Zurich between 2017 and 2020. The choice of the department depended on referrals from other hospitals and on the triage in the emergency unit. In total 28 ENT patients and 21 OMF patients were assessed. The following patients had to be excluded; two ENT patients because they did not answer our second phone call, two because they already underwent previous nasal surgery and one due to his or her age. The patient of the OMF department, who was excluded, did not answer our second phone call. The study population finally consisted of $23 \mathrm{ENT} / \mathrm{non}-\mathrm{CBCT}$ patients and $20 \mathrm{OMF/CBCT}$ patients.

Our other inclusion criteria were age between 18 and 70 years, a minimum of six months past surgery and a signed general consent for further processing of health-related personal details and biological material for research. Our other exclusion criteria were endonasal neoplasia, septal hematoma or combination of
Table 1. Comparison of the different splinting protocols between the two departments.

\begin{tabular}{|lcc|}
\hline Splinting protocol & $\begin{array}{l}\text { ENT department } \\
\text { (number of pa- } \\
\text { tients) }\end{array}$ & $\begin{array}{l}\text { OMF department } \\
\text { (number of pa- } \\
\text { tients) }\end{array}$ \\
\hline $\begin{array}{l}\text { Doyle Splints + endonasal } \\
\text { packing (Tampograss }{ }^{\oplus} \text { ) } \\
\text { nasal cast }\end{array}$ & 0 & 16 \\
\hline Doyle Splints + nasal cast & 0 & 1 \\
\hline Tampograss ${ }^{\circledR}+$ nasal cast & 0 & 2 \\
\hline Spongostan ${ }^{\circledR}+$ nasal cast & 3 & 0 \\
\hline Nasal cast only & 19 & 1 \\
\hline No packing or cast & 1 (cast refusal) & 0 \\
\hline Total & 23 & 20 \\
\hline
\end{tabular}

the nasal fracture with other midfacial fractures. Patients with a fracture of the mandibular bone still got included in the study, because midfacial fractures do not include fractures of the mandibular bone. Patients were recruited from the surgical planning tool of the clinical information system and the patients complying with the in- and exclusion criteria were contacted directly.

\section{Surgical procedure}

The surgeries at the ENT department were performed by either fully trained surgeons or by trainees with the supervision of a board-certified ENT surgeon. Reduction was performed in a closed manner using a bone elevator and manual pressure. All patients, except for one who refused it, received a thermoplastic cast for one week. In three patients with very mobile fragments internal splinting using resorbable packing material (Spongo$\operatorname{stan}^{\oplus}$ ) was used. At the ENT department a nasal reduction is performed within ten days after fracture; immediately when there is no soft tissue oedema or after the reduction of the swelling. At the OMF department closed nasal reductions were also performed by different surgeons, either by consultants or residents under consultant's supervision. In analogy to the ENT department, reduction was performed using a bone elevator and manual pressure. Generally, the intervention was performed within seven days post trauma in general anaesthesia. In 16 cases of mobile fragments both Doyle splints and endonasal packing were applied after reduction; in three patients either Doyle splints or nasal packing were applied. Table 1 shows a comparison of splinting protocols between the two departments. At the end of the procedure 3D imaging was conducted in a prone position for quality control, (xCAT, Xoran Technologies, Ann Arbor, MI, USA) either before or shortly after the thermoplastic cast had been applied. Multiplanar image reconstructions in natural head position (NHP) were used to analyse correct bone reduction and if necessary, instant corrections were performed. Endonasal splints were removed five days postoperatively. Figure 1 shows 

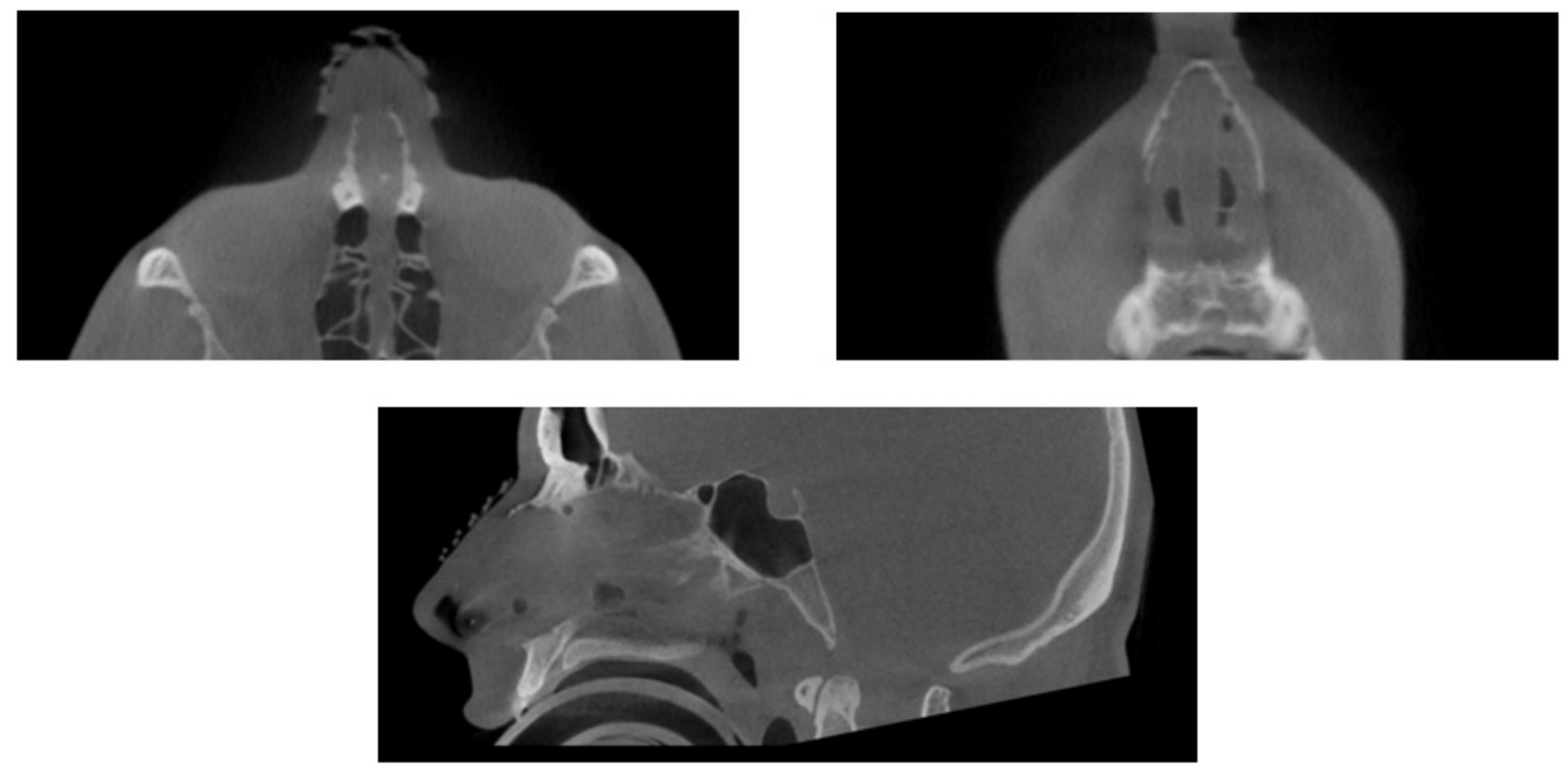

Figure 1. Multiplanar image reconstruction in natural head position (NHP) of an intraoperative CBCT picture showing a satisfactory result.

an intraoperative CBCT picture as an example.

\section{Data collection}

All data was collected on the phone or by consulting the patient's personal medical history. During the second phone call, subjective data was collected by a trained examiner (C.B.), assessing patient satisfaction with aesthetic and functional aspects of the nose. The subjective data was collected by using the Standardized Cosmesis and Health Nasal Outcomes Survey (SCHNOS) score ${ }^{(8)}$.

The SCHNOS ${ }^{(8)}$ score is a 10 -item measure to assess aesthetic and also functional aspects of the nose ${ }^{(9)}$. The SCHNOS score contains four questions assessing the function of the nose, five questions assessing the aesthetics of the nose and one question assessing the mood and self-esteem in regards to the nose. For each of the ten questions participants had to provide a value between 0 (no problem) and 5 (extreme problem). The SCHNOS score is divided in to two subscales; the "obstruction score" (SCHNOS-O), containing the questions 1-4 and the "cosmesis score" (SCHNOS-C), containing the questions 5-10. For the analysis of the SCHNOS-O score, the total score of the questions $1-4$ is divided by 20 and is multiplied by 100 . For the analysis of the SCHNOS-C score, the total score of the questions 5-10 is divided by 30 and multiplied by 100 (10). Consequently, the maximum attainable SCHNOS-O and SCHNOS-C score is 100 and the maximum attainable total SCHNOS score is 200. The minimal clinically important difference (MCID) is an important value in medical research to assess whether a change on a numerical scale is not only statistically significant, but also clinically meaningful to patients ${ }^{(11)}$. The MCID is 28.3 for the SCHNOS-O score and 18.0 for the SCHNOS-C score ${ }^{(10)}$.

After completion of the SCHNOS questionnaire, participants were asked about their subjective assessment of the aesthetics and function prior to and after the nasal fracture, with the aim of detecting patients with pre-existing impairments of either the aesthetics or function of their nose. The responses were classified as better, unchanged or worse. Furthermore, participants were asked whether they had already undergone or wish to undergo revision surgery.

We noticed, that patients from the OMF/CBCT group often underwent closed nasal reduction in combination with additional surgical procedures. These additional procedures made a comparison of the surgery duration with a statistical test impossible. Therefore, we randomly picked four patients without additional surgical procedures from each group to compare the surgery duration.

\section{Statistical analysis}

All statistical analyses were performed with an a-error of $5 \%$, using the statistical software IBM SPSS Statistics (Version 26.0.0.0 64-Bit, USA). All reported p-values are two-tailed. Graphic data presentation was performed using GraphPad Prism (Version 8.0.0 64-Bit, USA). Age was considered as continuous data and presented as mean \pm standard deviation (SD). Total SCHNOS score, SCHNOS-O and SCHNOS-C, gender, rate of revision surgery and aesthetics and function before and after the accident were considered as categorical variables and presented as median with an interquartile range (IQR). The normality of distribution was tested by using the Shapiro-Wilk Test. Comparisons of continuous variables with a normal distribution were performed 
A

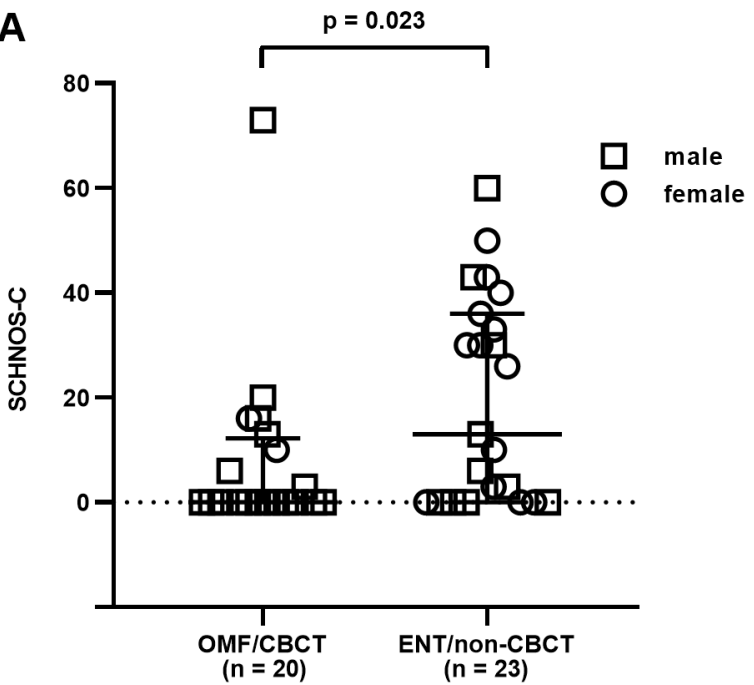

B

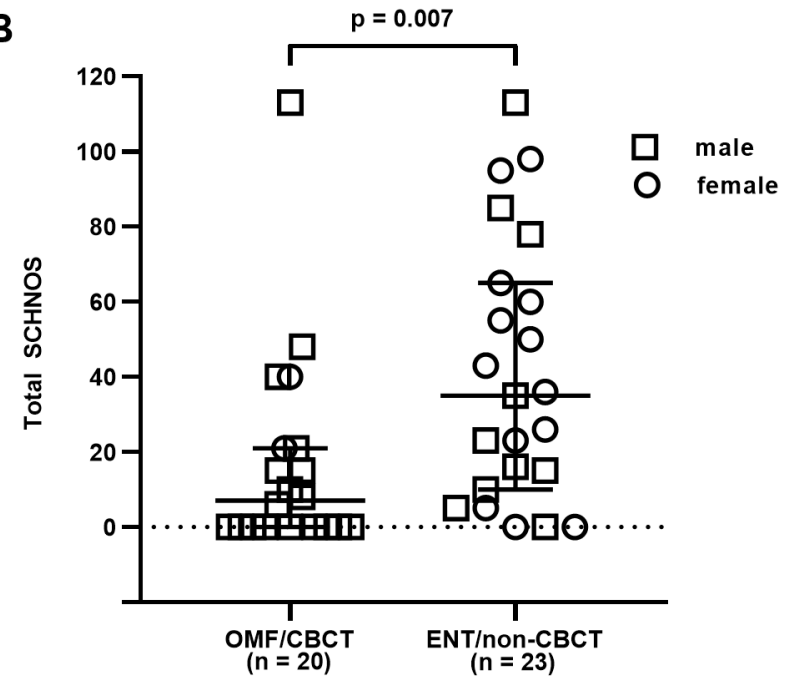

Figure 2. Comparison of SCHNOS-C (A) and total SCHNOS (B) between patients of the OMF/CBCT group and patients of the ENT/non-CBCT group.

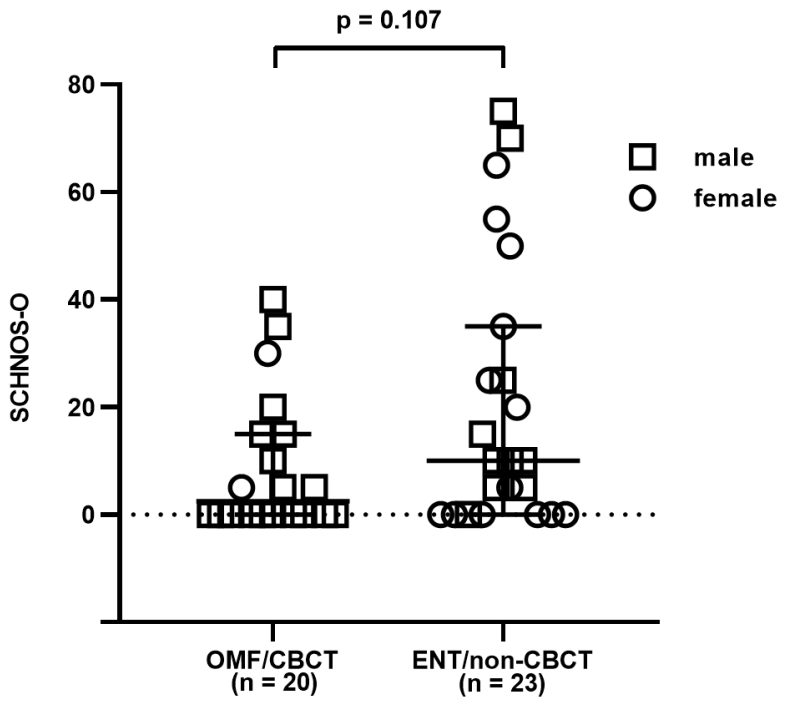

Figure 3. Comparison of SCHNOS-O score between patients of the OMF/ $\mathrm{CBCT}$ group and patients of the ENT/non-CBCT group.

using a Student t-Test. For comparisons of continuous variables with a non-normal distribution and for comparisons between two categorical variables with an ordinal scale, a Wilcoxon Test was used. For comparisons of categorical variables, using a cross table with values below five, a Fisher's Exact Test was used. Correlations between categorical data with an ordinal scale were calculated using a Spearman Rank Correlation. In the end a posthoc power analysis was performed using an online power and sample size calculator (https://www.sealedenvelope.com/power/continuous-superiority, United Kingdom).

\section{Results}

The male to female ratio in ENT/non-CBCT patients was 10:13; that in OMF/CBCT patients was 18:2. The average age in the
ENT/non-CBCT group was $39.57 \pm 12.31$ years and $40.25 \pm 14.55$ years in the OMF/CBCT group. Table 2 summarizes demographic factors, mean and median values of all our measurements for each group.

As Figure 2 demonstrates, the comparison of SCHNOS questionnaires between the two groups revealed significantly higher SCHNOS-C (A) (Wilcoxon Test: $p=0.023$ ) and total SCHNOS (B) (Wilcoxon Test: $p=0.007$ ) scores in the ENT/non-CBCT group. As Figure 3 demonstrates, the comparison of SCHNOS-O scores between the two groups showed no significant difference (Wilcoxon Test: $p=0.107$ ). The same comparison of SCHNOS values was performed for only the male subjects of both groups, revealing no significant difference in SCHNOS-C (Figure 4A) (Wilcoxon Test: $p=0.226$ ) and a trend to higher SCHNOS-O scores in the ENT/non-CBCT group (Wilcoxon Test: $p=0.051$ ). The comparison of SCHNOS scores between male and female subjects from both groups combined showed significantly higher median SCHNOS-C (Figure 4B) (Wilcoxon Test: $\mathrm{p}=0.013$ ) and total SCHNOS (Wilcoxon Test: $p=0.025$ ) scores in female subjects, but no difference for SCHNOS-O scores (Wilcoxon Test: $p=0.571$ ). Comparing the desire for revision surgery (Fisher's Exact Test: $p=0.076$ ), aesthetics (Wilcoxon test: $p=0.678$ ) and function (Wilcoxon test: $p=0.215$ ) prior to and after the accident, no significant difference could be demonstrated between the two groups. Comparing the desire for revision surgery there was no difference between female and male subjects (Fisher's Exact Test: $p=0.281$ ). Table 3 summarizes the values and differences between male and female subjects.

As Figure 5 demonstrates, the time between the accident and closed reduction showed a significant difference between the two groups, with a mean of $7.52 \pm 2.35$ days in the ENT/ non-CBCT group and a mean of $3.5 \pm 2.63$ days in the OMF/ CBCT group (Wilcoxon Test: $p=<0.0001$ ). Correlations of time 

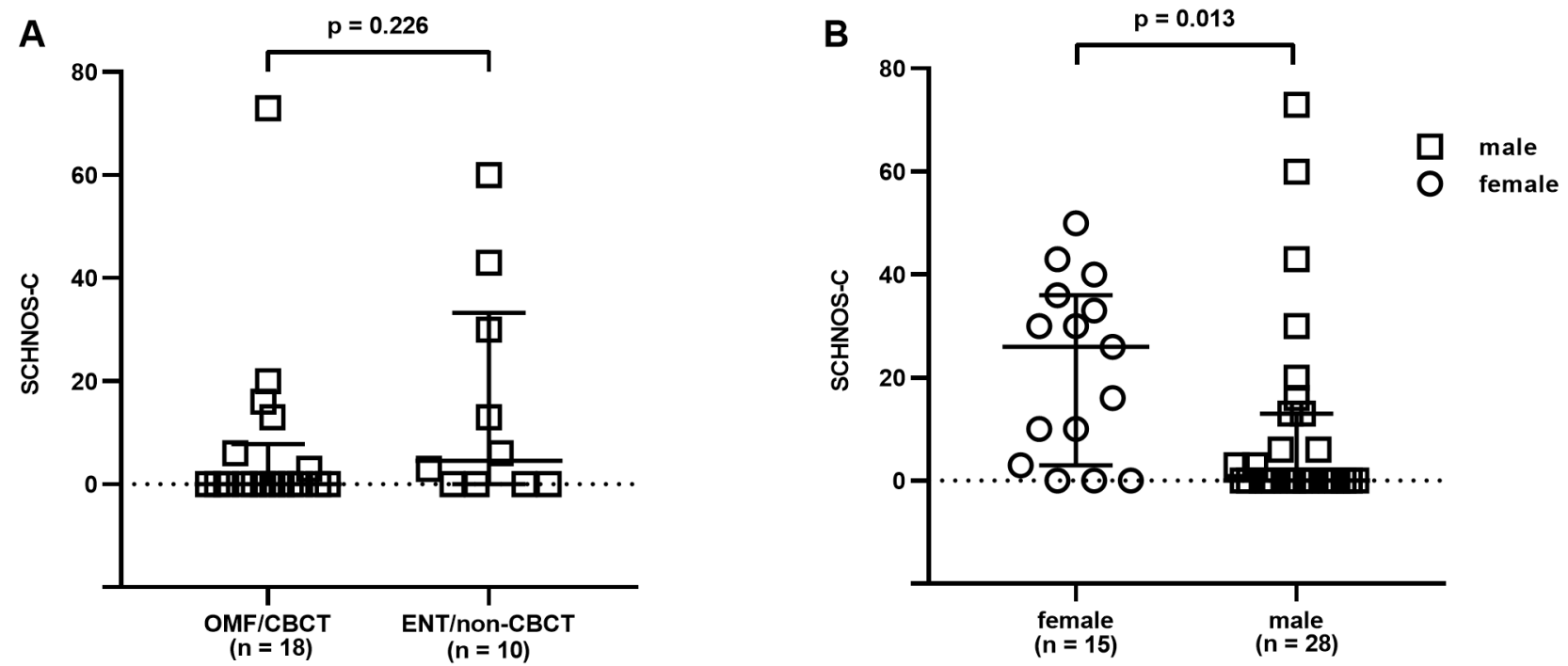

Figure 4. Comparison of SCHNOS-C between male patients of the two groups (A) and between male and female patients of both groups (B).

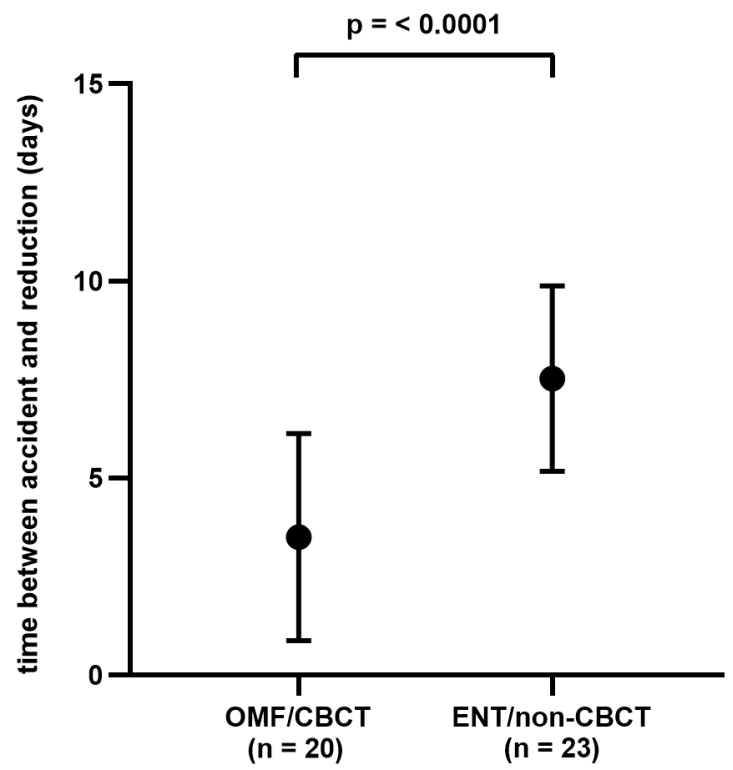

Figure 5. Comparison of the elapsed time between the accident and the closed reduction of the nose between the OMF/CBCT group and the ENT/non-CBCT group.

between accident and reduction with SCHNOS-O, SCHNOS-C, total SCHNOS and aesthetics and function before and after the accident showed no statistical significance.

A significant, negative correlation of SCHNOS-C with aesthetics before and after the accident (Spearman Rank Correlation: $r=$ $-0.69, p=<0.001$ ), as well as SCHNOS-O with function before and after the accident (Spearman Rank Correlation: $r=-0.44, p=$ 0.003 ) could be demonstrated.

Comparing the samples of four randomly picked patients, a longer duration of nasal reduction could be demonstrated in the OMF group with a mean of 19.5 minutes compared to the ENT group with a mean of 12 minutes.
The posthoc power analysis with a significance level of $5 \%$, a power of $80 \%$ and median values of SCHNOS-C of the two groups revealed a required sample size of 21 patients per group and total sample size of 42 patients.

\section{Discussion}

In the present study, our aim was to investigate the effect of intraoperative $\mathrm{CBCT}$ imaging on patient satisfaction after closed nasal reduction. Nasal reductions with and without intraoperative CBCT imaging have never before been compared to each other regarding patient satisfaction with postoperative aesthetic and functional aspects of the nose.

Wild et al. suggested subjective patient satisfaction be the most important outcome measure after closed reduction of the nose (12). As there are still no objective measures to quantify success after closed nasal reduction, we used the subjective SCHNOS score to measure patient satisfaction. The significant, negative correlations of SCHNOS-C scores with aesthetics and SCHNOS$\mathrm{O}$ scores with function prior to and after the accident indicate that the SCHNOS score appropriately measures aesthetic and functional aspects of the nose and is an adequate measure for our study.

Hung et al. also assessed patient satisfaction after closed nasal reduction. They demonstrated a dissatisfaction rate of $24 \%$ with nasal aesthetics and a dissatisfaction rate of $21 \%$ with nasal patency. $29 \%$ indicated that they would like to undergo revision surgery. In our study the dissatisfaction rate with nasal aesthetics and function and the wish for revision surgery were higher in the ENT/non-CBCT patients and lower in the OMF/CBCT patients compared to Hung et al. ${ }^{(13)}$.

The comparison of SCHNOS-O scores, representing functional outcome showed no difference between the two groups. The comparison of SCHNOS-C scores showed significantly lower 
Table 2. Comparison of the measurements between ENT patients treated without intraoperative CBCT and OMF patients treated with intraoperative CBCT.

\begin{tabular}{llll}
\hline Characteristics & ENT/non-CBCT & \multicolumn{1}{c}{ OMF/CBCT } & P Value \\
\hline Male/female & $10 / 13$ & $18 / 2$ & 0.002 \\
\hline Mean age (y) & $39.57 \pm 12.31$ & $40.25 \pm 14.55$ & 0.942 \\
\hline $\begin{array}{l}\text { SCHNOS-O } \\
\text { (median, IQR) }\end{array}$ & 10,35 & $2.5,15$ & 0.107 \\
\hline $\begin{array}{l}\text { SCHNOS-C } \\
\text { (median, IQR) }\end{array}$ & 13,36 & $0,12.25$ & 0.023 \\
\hline $\begin{array}{l}\text { Total SCHNOS } \\
\text { (median, IQR) }\end{array}$ & 35,55 & 7,21 & 0.007 \\
\hline $\begin{array}{l}\text { Following rhinoplasty } \\
\text { (yes/no) }\end{array}$ & $1 / 23$ & $1 / 20$ & \\
\hline $\begin{array}{l}\text { Planned rhinoplasty } \\
\text { (\%) }\end{array}$ & 34.8 & & 0.076 \\
\hline $\begin{array}{l}\text { Aesthetics pre-/post } \\
\text { (\%) }\end{array}$ & $\begin{array}{l}34.8 \text { worse, } \\
52.2 \text { no change }\end{array}$ & $\begin{array}{l}21.7 \text { worse, } \\
56.5 \text { no change } \\
8.7 \text { better }\end{array}$ & 0.678 \\
\hline $\begin{array}{l}\text { Function pre-/post } \\
\text { (\%) }\end{array}$ & $\begin{array}{l}34.8 \text { worse, } \\
60.9 \text { no change }\end{array}$ & $\begin{array}{l}13 \text { worse, } \\
60.9 \text { no change } \\
13 \text { better }\end{array}$ & 0.215 \\
\hline $\begin{array}{l}\text { Time to reduction } \\
\text { (days, mean } \pm \text { SD) }\end{array}$ & \begin{tabular}{l}
7.3 better \\
\hline
\end{tabular} & $3.5 \pm 2.63$ & $<0.0001$ \\
\hline
\end{tabular}

scores for the OMF/CBCT group, indicating better aesthetic outcome in this group. Even though the difference between the two groups was statistically significant, the difference of 11.98 in the SCHNOS-C score is lower than the suggested MCID of 18 for SCHNOS-C score ${ }^{(10,11)}$. A difference below MCID means that a difference that may be statistically significant has no clinical importance (11).

Due to the retrospective study setup the two groups did not match for gender, resulting in a higher number of male subjects in the OMF/CBCT group. Therefore, we compared SCHNOS-C scores only between male subjects of the two groups and also between male and female subjects from both groups combined. We could find no difference in SCHNOS-C scores between male subjects of the two groups but a significant difference between male and female subjects. Both of these findings indicate the involvement of gender as a confounder in our results. We suggest that the difference of SCHNOS-C between the ENT/ non-CBCT and OMF/CBCT groups does not seem to represent a difference between the two groups and strategies of intraoperative imaging, but rather a difference in gender distribution of the two groups. Our results show that women seem to be less satisfied with postoperative nasal aesthetics than men, potentially leading to the better SCHNOS-C scores in the OMF/CBCT group, with a higher proportion of male subjects. A possible explanation for this finding is that women seem to be more sensitive and pay more attention to remaining nasal deformities
Table 3. Comparison of the measurements between female and male patients of both groups (ENT/non-CBCT and OMF/CBCT) together.

\begin{tabular}{|c|c|c|c|}
\hline Characteristics & Female & Male & P Value \\
\hline Mean age $(y)$ & $42.07 \pm 12.68$ & $38.71 \pm 13.60$ & 0.435 \\
\hline $\begin{array}{l}\text { SCHNOS-O } \\
\text { (median, IQR) }\end{array}$ & 5,35 & 5,15 & 0.571 \\
\hline $\begin{array}{l}\text { SCHNOS-C } \\
\text { (median, IQR) }\end{array}$ & 26,33 & 0,13 & 0.013 \\
\hline $\begin{array}{l}\text { Total SCHNOS } \\
\text { (median, IQR) }\end{array}$ & 40,39 & 10,32 & 0.025 \\
\hline $\begin{array}{l}\text { Following rhinoplasty } \\
\text { (yes/no) }\end{array}$ & $1 / 15$ & $1 / 28$ & \\
\hline $\begin{array}{l}\text { Planned rhinoplasty } \\
\text { (\%) }\end{array}$ & 33.3 & 17.9 & 0.001 \\
\hline $\begin{array}{l}\text { Aesthetics pre-/post } \\
\text { (\%) }\end{array}$ & $\begin{array}{l}53.3 \text { worse, } \\
33.3 \text { no change } \\
13.3 \text { better }\end{array}$ & $\begin{array}{l}17.9 \text { worse, } \\
71.4 \text { no change } \\
10.7 \text { better }\end{array}$ & 0.073 \\
\hline $\begin{array}{l}\text { Function pre-/post } \\
\text { (\%) }\end{array}$ & $\begin{array}{l}26.7 \text { worse } \\
73.3 \text { no change } \\
0 \text { better }\end{array}$ & $\begin{array}{l}25 \text { worse } \\
60.7 \text { no change } \\
14.3 \text { better }\end{array}$ & 0.440 \\
\hline
\end{tabular}

after closed nasal reduction than men. Studies about patient satisfaction after rhinoplasty demonstrated either no difference between male or female subjects ${ }^{(14)}$ or lower satisfaction rates in male subjects ${ }^{(15)}$. Interestingly studies about patient satisfaction after nasal fractures showed a trend to higher satisfaction rates in male subjects ${ }^{(16)}$, compared to female subjects and a higher risk of corrective septorhinoplasty in female subjects ${ }^{(17,18)}$. Our results demonstrated as expected, a significant difference in the timing of the reduction after nasal fracture between the two groups. There are different opinions on the appropriate timing of nasal reduction after nasal fracture ${ }^{(4)}$. Some injuries accompanying nasal fracture, such as septal hematoma require immediate surgery. In contrast to that, a soft tissue oedema accompanying nasal fracture makes immediate closed nasal reduction difficult and therefore many patients should be reevaluated no earlier than three to four days after the accident (19). Some authors suggest treatment of nasal fractures within no more than two weeks after the accident ${ }^{(20,21)}$; other authors suggest a treatment within no more than 10 days ${ }^{(19,22,23)}$. Comparing the ENT and OMF departments at the University Hospital of Zurich different approaches regarding the timing of closed nasal reduction after nasal fracture can be found. The mean time from accident to reduction is higher in patients treated at the ENT department because it focuses on the reduction of the soft tissue oedema, with the aim of having a better view on the operating area. The mean time from accident to reduction is lower in patients treated at the OMF department because it focuses on preventing greater bone healing prior to surgery and as a consequence thereof more osteotomies. The lack of correlation of 
the elapsed time with functional and aesthetic outcome and the recommendation of treatment within 10-14 days ${ }^{(19-23)}$ indicate that both strategies have their justification.

The comparison of the four randomly picked patients of each group revealed a slightly longer duration of nasal reduction in the OMF/CBCT group compared to the ENT/non-CBCT group. However, the immediate $C B C T$ feedback on the positioning of the nasal bony elements is a pleasant feature for surgeons and allows instant corrections of an unfavourable positioning.

\section{Conclusion}

This is the first study to investigate subjective outcome of closed nasal reduction with and without intraoperative $\mathrm{CBCT}$ imaging. We could not find a clinically important benefit from one strategy over the other. The results must be considered with some caution, due to the potential confounding factor of gender in our study population. Female subjects seem to be less satisfied with the aesthetics of their nose postoperatively, potentially being more sensitive to remaining nasal deformities, compared to male subjects.

\section{List of abbreviations}

CBCT: Cone-Beam Computed Tomography; IQR: interquartile range; MCID: minimal clinically important difference; NHP: natural head position; SCHNOS: Standardized Cosmesis and Health Nasal Outcomes Survey; SCHNOS-C: Cosmesis score of the Standardized Cosmesis and Health Nasal Outcomes Survey;
SCHNOS-O: Obstruction score of the Standardized Cosmesis and Health Nasal Outcomes Survey.

\section{Authorship contribution}

$C B$ recruited the patients, collected the data, performed statistical testing and wrote the manuscript. MB searched the clinical information system for eligible OMF patients and reviewed the manuscript. MBS planned, designed and supervised the study and reviewed the manuscript.

\section{Acknowledgments}

Not applicable.

\section{Funding}

Not applicable.

\section{Ethics approval and consent to participate}

Not applicable.

\section{Consent for publication}

Not applicable.

\author{
Availability of data and materials \\ Not applicable.
}

\section{Conflict of interest}

There are no conflicts of interest.

\section{References}

1. Lu GN, Humphrey CD and Kriet JD. Correction of Nasal Fractures. Facial Plast Surg Clin North Am 2017; 25: 537-546.

2. VandeGriend ZP, Hashemi A and Shkoukani M. Changing trends in adult facial trauma epidemiology. J Craniofac Surg 2015; 26: 108-112.

3. Lascaratos JG, Segas JV, Trompoukis CC, et al. From the roots of rhinology: the reconstruction of nasal injuries by Hippocrates. Ann Otol Rhinol Laryngol 2003; 112: 159162.

4. Mondin V, Rinaldo $A$ and Ferlito $A$. Management of nasal bone fractures. Am J Otolaryngol 2005; 26: 181-185.

5. Bremke M, Wiegand S, Sesterhenn AM, et al Digital volume tomography in the diagnosis of nasal bone fractures. Rhinology 2009; 47: 126-131.

6. Bremke M, Leppek R and Werner JA. [Digita volume tomography in ENT medicine]. HNO 2010; 58: 823-832.

7. Lee IS, Lee JH, Woo CK, et al Ultrasonography in the diagnosis of nasal bone fractures: a comparison with conventional radiography and computed tomography. Eur Arch Otorhinolaryngol 2016; 273 413-418. 2015/03/08.
8. Moubayed SP, loannidis JPA, Saltychev M, et al. The 10-Item Standardized Cosmesis and Health Nasal Outcomes Survey (SCHNOS) for Functional and Cosmetic Rhinoplasty. JAMA Facial Plast Surg 2018; 20: 37-42.

9. van Zijl FVWJ, Mokkink LB, Haagsma JA, et al. Evaluation of Measurement Properties of Patient-Reported Outcome Measures After Rhinoplasty: A Systematic Review. JAMA Facial Plast Surg 2019; 21: 152-162.

10. Kandathil CK, Saltychev M, Abdelwahab $M$, et al. Minimal Clinically Important Difference of the Standardized Cosmesis and Health Nasal Outcomes Survey. Aesthet Surg J 2019; 39: 837-840.

11. McGlothlin AE and Lewis RJ. Minimal clinically important difference: defining what really matters to patients. JAMA 2014; 312 : 1342-1343.

12. Wild DC, El Alami MA and Conboy PJ. Reduction of nasal fractures under local anaesthesia: an acceptable practice? Surgeon 2003; 1: 45-47.

13. Hung T, Chang W, Vlantis AC, et al. Patient satisfaction after closed reduction of nasal fractures. Arch Facial Plast Surg 2007; 9: 40-43.

14. Koybasi S, Bicer YO, Seyhan S, et al. Satisfaction in rhinoplasty: the possible impact of anxiety and functional outcome. Eur Arch Otorhinolaryngol 2018; 275: 729733. 2018/01/12.

15. Khansa I, Khansa $L$ and Pearson GD. Patient Satisfaction After Rhinoplasty: A Social Media Analysis. Aesthet Surg J 2016; 36: NP1-5. 2015/06/10.

16. Basheeth N, Donnelly M, David S, et al. Acute nasal fracture management: A prospective study and literature review. Laryngoscope 2015; 125: 2677-2684.

17. Li K, Moubayed SP, Spataro E, et al. Risk Factors for Corrective Septorhinoplasty Associated With Initial Treatment of Isolated Nasal Fracture. JAMA Facial Plast Surg 2018; 20: 460-467.

18. Spataro E, Piccirillo JF, Kallogjeri D, et al. Revision Rates and Risk Factors of 175842 Patients Undergoing Septorhinoplasty. JAMA Facial Plast Surg 2016; 18: 212-219.

19. Rubinstein B and Strong EB. Management of nasal fractures. Arch Fam Med 2000; 9: 738-742.

20. Staffel JG. Optimizing treatment of nasal fractures. Laryngoscope 2002; 112: 17091719.

21. Sharma SD, Kwame I and Almeyda J. Patient aesthetic satisfaction with timing of nasal fracture manipulation. Surg Res Pract 2014; 
2014: 238520. 2014/01/02.

22. Rohrich RJ and Adams WP. Nasal fracture management: minimizing secondary nasal deformities. Plast Reconstr Surg 2000; 106: 266-273.

23. Ridder GJ, Boedeker CC, Fradis $M$, et al. Technique and timing for closed reduction of isolated nasal fractures: a retrospective study. Ear Nose Throat J 2002; 81: 49-54.
Michael Soyka, MD

UniversitätsSpital Zürich

Klinik für Ohren-, Nasen-, Hals- und

Gesichtschirurgie

Frauenklinikstrasse 24

$\mathrm{CH}-8091$ Zürich

Switzerland

Tel: +41-44-255-1111

E-mail: michael.soyka@usz.ch

ISSN: 2589-5613 / O2021 The Author(s). This work is licensed under a Creative Commons Attribution 4.0 International License. The images or other third party material in this article are included in the article's Creative Commons license, unless indicated otherwise in the credit line; if the material is not included under the Creative Commons license, users will need to obtain permission from the license holder to reproduce the material. To view a copy of this license, visit http://creativecommons.org/licenses/by/4.0/ 